\title{
Calcul du champ d'écoulement dans une turbine Kaplan et calcul des contraintes dans les pales
}

\author{
Calculation of the flow field in a Kaplan turbine \\ and of the stresses in the blades
}

\author{
I. Ancona et A. Comte
}

E.D.F. - D.E.R. Chatou

\section{Introduction}

L'étude complète d'une turbine hydraulique présente deux aspects :

- l'étude hydro-dynamique de la turbine permettant de déterminer les caractéristiques globales de la machine (puissance fournie, rendement . . .) et le comportement local du fluide, en particulier son adaptation (risques de cavitation, d'érosion, de vibrations d'origine hydrodynamique ...);

- l'étude mécanique des pièces constitutives de la turbine permettant de vérifier la bonne résistance aux efforts exercés (pression, rotation ....) dans toutes les plages de fonctionnement.

Cette deuxième étude est complémentaire de la première puisqu'elle nécessite une connaissance fine des caractéristiques de l'écoulement aussi bien dans les zones hors grilles que dans les aubages fixes et mobiles.

Un calcul quasi tridimensionnel de l'écoulement est mené à travers la turbine de l'amont des étages distributeurs (sortie de la volute) à l'aval de la roue mobile afin de déterminer ces caractéristiques.

La répartition du champ de pression statique sur les aubages mobiles est alors utilisée pour le calcul des efforts et des contraintes qui s'y exercent.

Une description de ces deux calculs, appliqués à une turbine de type KAPLAN, est donnée et les résultats sont présentés et comparés à des valeurs expérimentales acquises sur maquette pour la partie hydrodynamique et sur une turbine réelle pour la partie mécanique.

\section{Description de la méthode utilisée pour le calcul de l'écoulement}

- Principe de la méthode des deux surfaces (développée

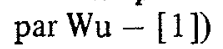

$\mathrm{Au}$ lieu de résoudre le problème tridimensionnel dans son entier, cette méthode résoud de façon itérative deux problèmes bidimensionnels qui sont définis sur deux familles de surfaces sécantes :

- $S_{1}$ : surface aube à aube ;

- $S_{2}$ : surface méridienne.

Les problèmes sont couplés : les résultats issus d'un calcul sont in troduits en tant que données dans l'autre et vice-versa - et ce jusqu'à convergence.

\section{- Hypothèses :}

L'écoulement est supposé incompressible et permanent dans le repère considéré.

Le fluide est considéré comme parfait.

(les effets de la viscosité pourront être pris en compte par l'intermédiaire d'un calcul de couche limite par la suite).

\section{- Calcul de l'écoulement méridien :}

Il nécessite quelques hypothèses complémentaires :

- l'écoulement est axisymétrique : on calcule un écoulement moyen correspondant à un nombre d'aubes infini.

- l'interaction entre le fluide et les aubes est prise en compte de la manière suivante :

- la vitesse (dans le repère considéré) est tangente à la surface squelette des profils.

- les forces exercées par le fluide sur les aubes sont perpendiculaires à ces dernières.

- le repère considéré est le repère absolu dans le distributeur et en dehors des roues et le repère relatif dans la roue mobile. Le système de coordonnées cylindriques est utilisé.

Ces hypothèses sont introduites dans les équations de l'écoulement qui peuvent se réduire finalement à une équation aux dérivées partielles du second degré, non linéaire, de la fonction de courant. La résolution de cette équation se fait à l'aide d'une méthode itérative à deux niveaux du fait de la non linéarité.

LA HOUILLE BLANCHE/N ${ }^{\circ} 7 / 8-1982$ 


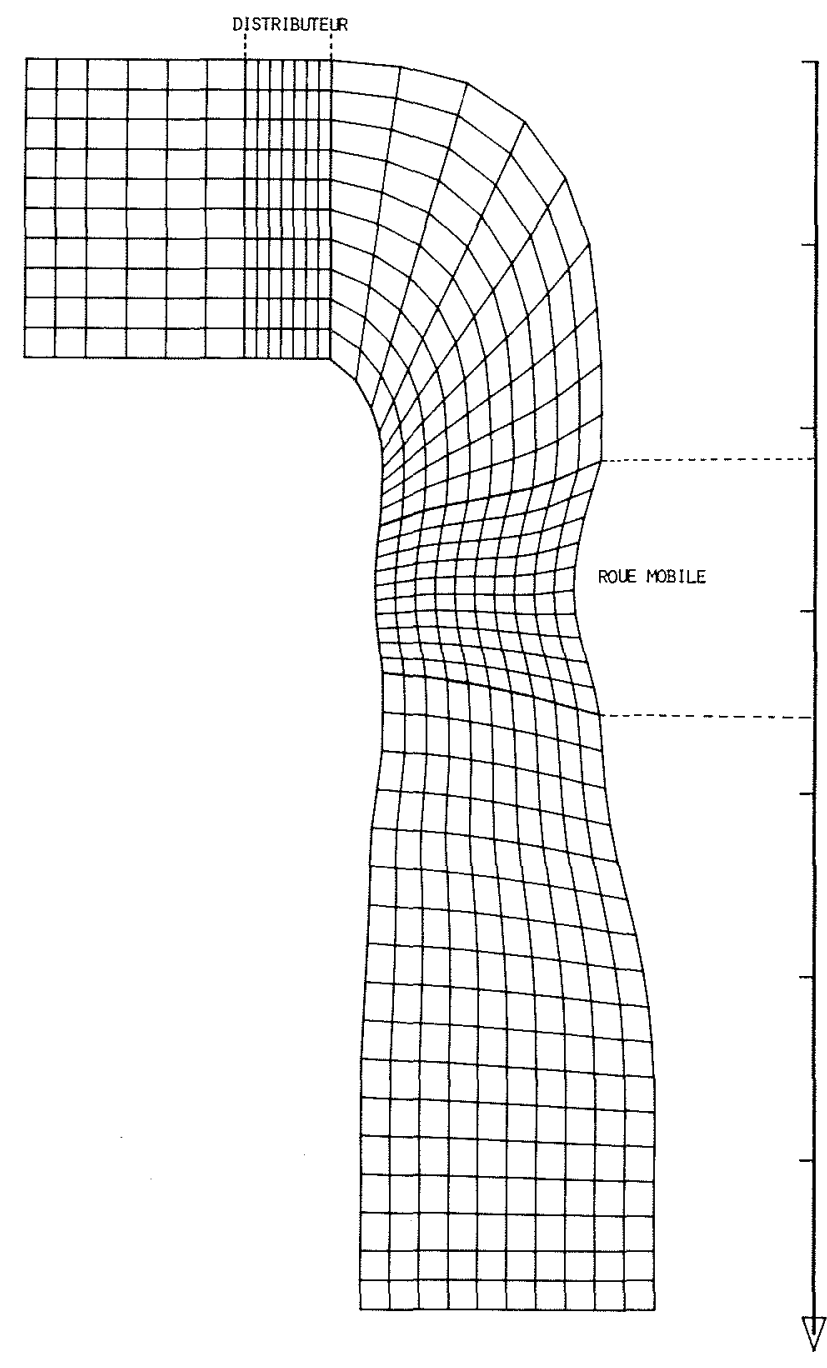

Figure 1 - Maillage d'une surface $S_{2}$.

Pour la discrétisation, un maillage du type de celui représenté sur la figure 1 et un schéma de différences finies centrées à neuf points sont utilisés.

Le calcul méridien fournit les résultats suivants :

- toutes les caractéristiques de l'écoulement (vitesses, pressions, angles ....) en chaque point du maillage.

- la géométrie et l'épaisseur des tubes de courant.

- l'angle d'attaque de l'écoulement à l'entrée de la roue mobile.

- la puissance fournie.

\section{Interface $S_{2}-S_{1}$ :}

Les surfaces $S_{1}$ sont générées à partir des lignes de courant du plan méridien par une rotation autour de l'axe. Elles sont donc axisymétriques.

Le calcul aube à aube est fait sur cinq surfaces $S_{1}$.

Le programme d'interface détermine la trace de l'aubage sur ces surfaces et prépare les données du programme aube à aube.

\section{Calcul aube à aube}

La méthode utilisée pour calculer l'écoulement sur la surface $S_{1}$ est celle de Katsanis [2].

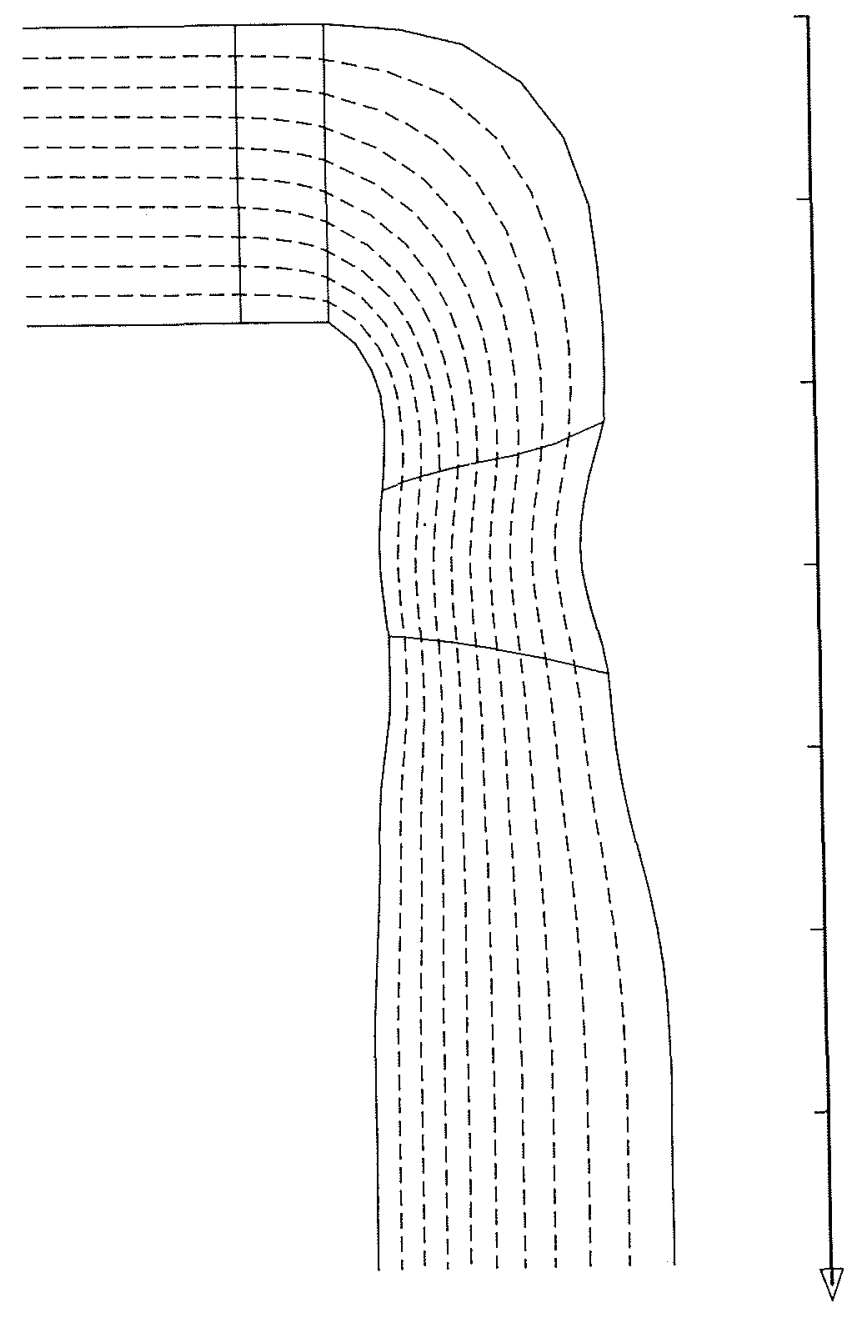

Figure 2 - Lignes de courant méridiennes.

\section{- Hypothèses}

- le repère utilisé est le repère relatif.

- l'écoulement est irrotationnel

- il est calculé dans le canal interaube sur une surface de courant axisymétrique.

- les vitesses sont uniformes dans la section amont.

- la condition de Kutta Joukowski fixe la circulation à l'aval de l'aubage mobile.

Les équations de l'écoulement peuvent être ramenées à une équation linéaire (du fait du caractère incompressible de l'écoulement) de la fonction de courant.

Un schéma de différences finies centrées et une méthode itérative sont utilisés pour la résolution de cette équation.

La réalisation de la condition de Kutta-Joukowsky nécessite un second niveau d'itérations : l'angle de sortie de l'écoulement est modifié jusqu'à ce que la vitesse ait la même valeur en deux points situés sur l'extrados et l'intrados de l'aubage au voisinage du bord de fuite. Les résultats issus du calcul aube à aube sont les suivants :

- l'écart flux-profil à la sortie de l'aubage,

- le profil des vitesses et la distribution des pressions en 
tout point du maillage de $S_{1}$ et en particulier sur l'extrados et l'intrados des aubages.

- les lignes de courant sur la surface $S_{1}$ entre deux aubes.

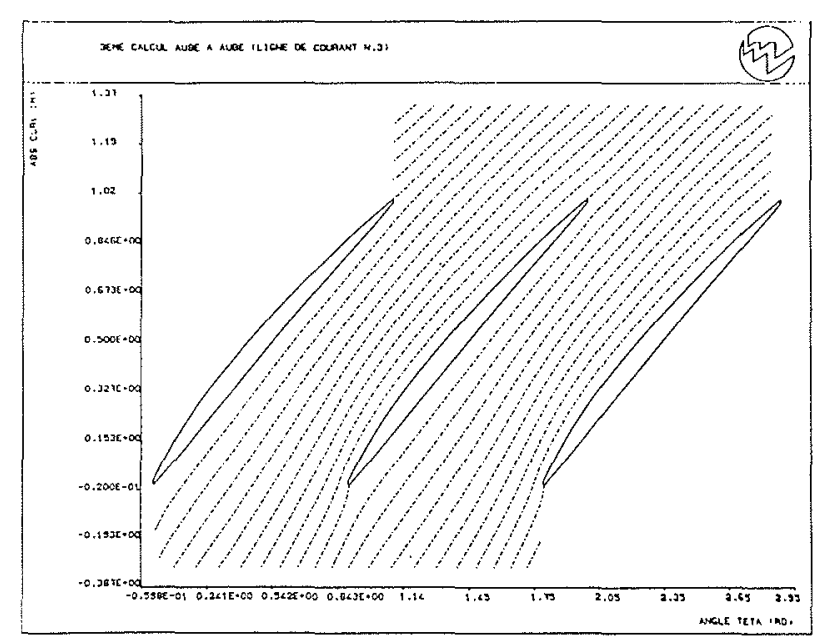

Figure 3 - Lignes de courant circonférentielles à l'issue du $3^{\mathrm{e}}$ calcul aube à aube (Ligne de courant $\mathrm{n}^{\circ} 3$ ).
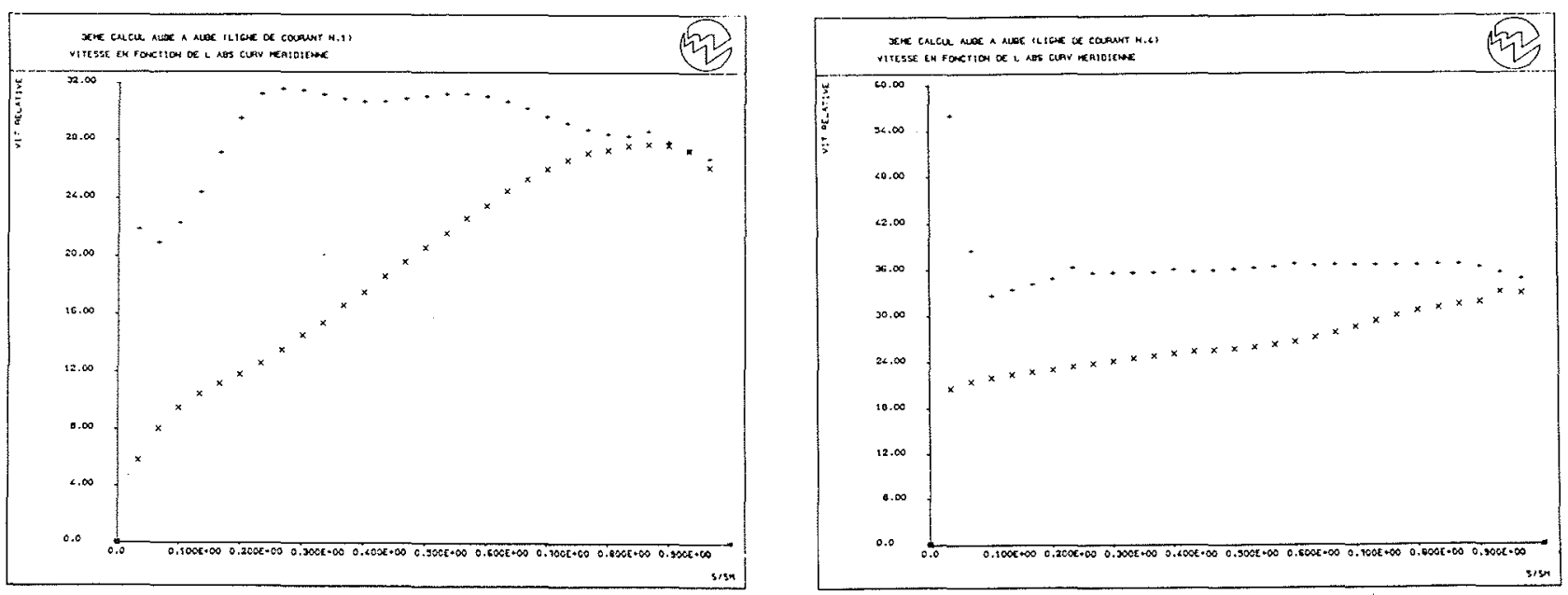

Figure $4 \mathrm{a}$ - Répartition des vitesses relatives en fonction de l'abscisse curviligne méridienne. (Ligne de courant $\mathrm{n}^{\circ} 1-$ Ligne de courant $\mathrm{n}^{\circ} 4$ ) (Extrados-Intrados) à l'issue du $3^{\mathrm{e}}$ calcul aube à aube.
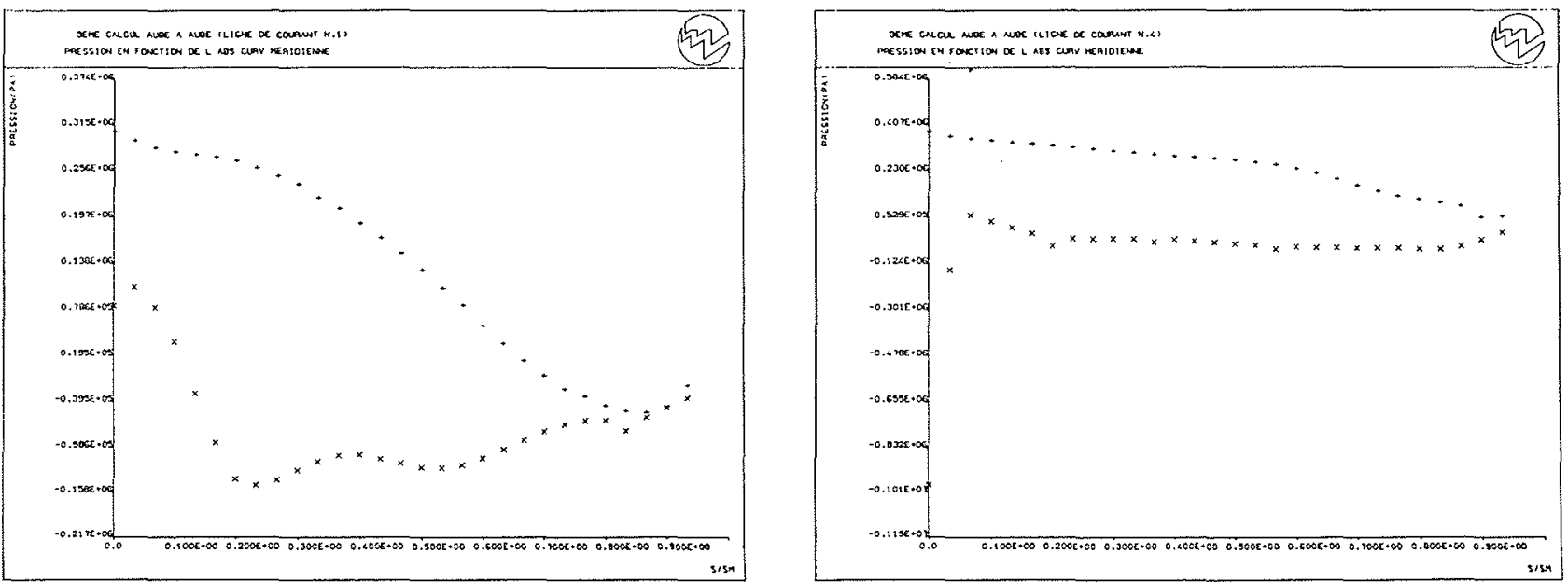

Figure $4 \mathrm{~b}-$ Répartition des pressions en fonction de l'abscisse curviligne méridienne. (Ligne de courant $n^{\circ} 1-$ Ligne de courant $n^{\circ} 4$ (Extrados-Intrados) à l'issue du $3^{\mathrm{e}}$ calcul aube à aube. 


\section{Description de la turbine étudiée}

La turbine étudiée est une turbine hydraulique de type KAPLAN.

Comme la figure 5 l'indique, la turbine comporte :

- un distributeur (à entrée radiale) formé de deux rangées d'aubes : les avant-directrices - aubes fixes et les directrices - aubes orientables.

- une roue mobile axiale à calage variable - Son ouverture est fonction de celle du distributeur.

Les caractéristiques de la turbine sont les suivantes:

- vitesse de rotation $N=150 \mathrm{tr} / \mathrm{min}$

- hauteur $H_{n}=36 \mathrm{~m}$

- débit dans le cas considéré $Q=150 \mathrm{~m}^{3} / \mathrm{s}$

- puissance théorique dans le cas considéré $P_{t h}=53 \mathrm{MW}$

\section{Etude hydrodynamique}

\section{- Mesures expérimentales de l'écoulement :}

Les mesures ont été faites sur un modèle réduit de la turbine à l'échelle : $1 / 12^{\mathrm{e}}$.

Les sondages de vitesse ont été effectués en amont et en aval de la roue mobile à l'aide d'une sonde à cing trous. La position de la sonde (cf. fig. 5) à l'amont de la roue n'a pas permis le sondage sur toute la largeur du canal.

\section{- Calcul de l'écoulement :}

Pour le calcul méridien, on a considéré deux grilles : les directrices et la roue mobile.

Les avant-directrices ont été prises en compte par l'angle qu'elles imposent à l'écoulement à l'entrée des directrices.

Le couplage calcul méridien - calcul aube à aube a été fait pour la roue mobile. Il commence par un calcul méridien.

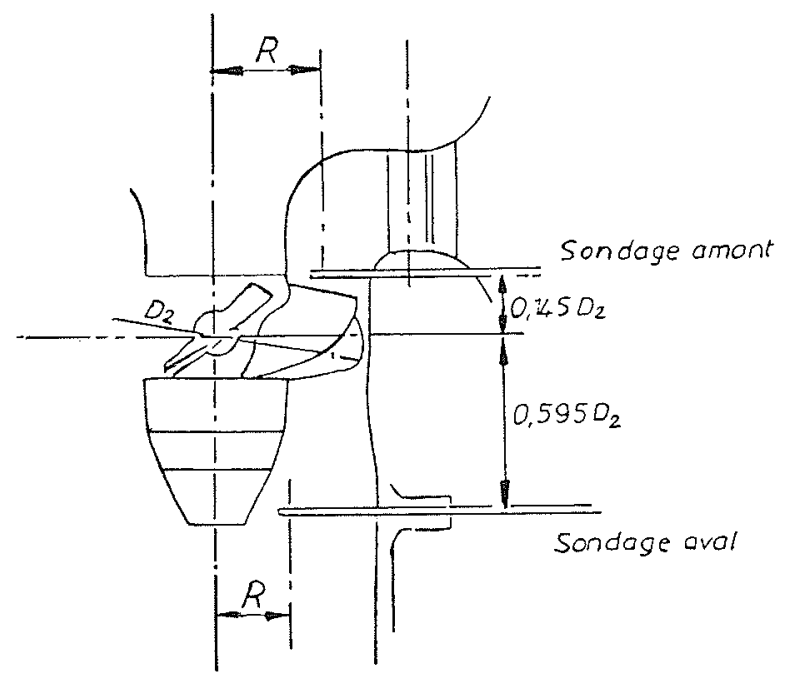

Figure 5 - Emplacement des sondages de vitesse.

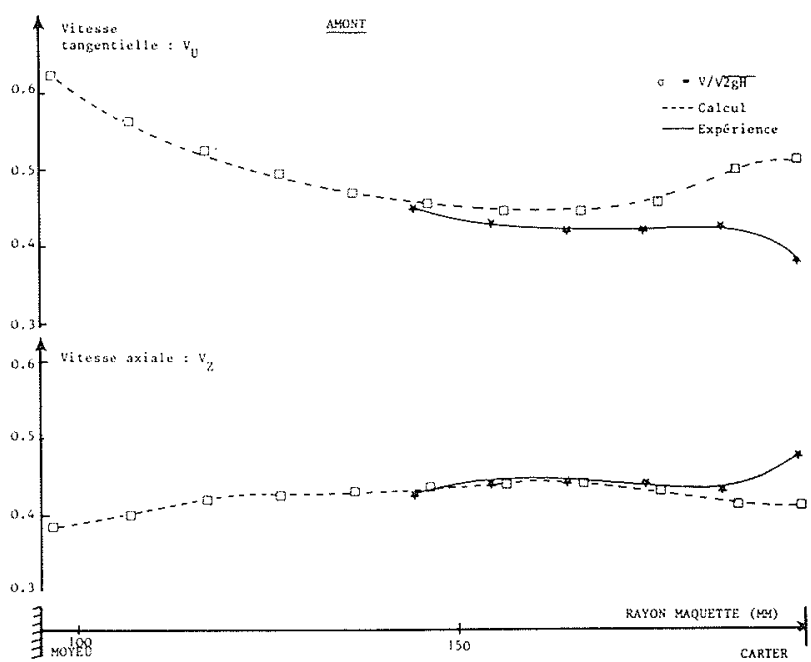

Figure 6 - Comparaison "Calcul-Expérience" à l'amont de la roue mobile.

Quelques résultats du calcul d'écoulement sont présentés:

- les lignes de courant de la surface $S_{2}$ (fig. 2)

- celles d'une surface $S_{1}$ (fig. 3)

- les répartitions de pressions et de vitesses sur l'extra. dos et sur l'intrados des aubes de deux surfaces $S_{1}$ (fig. 4).

Les conditions de fonctionnement paraissent assez bonnes, le fluide arrivant sur les aubes mobiles avec une légère incidence.

\section{- Comparaison des résultats théoriques et expéri- mentaux}

La figure 6 présente une comparaison calcul-expérience en amont de la roue mobile.

Les courbes montrent une bonne concordance au milieu de la veine.

La différence apparaissant au voisinage du carter peut être due à l'influence de la paroi (effets de couche limite et écoulements secondaires) qui n'est pas prise en compte dans le calcul.

Les valeurs dont on dispose à l'entrée de la roue mobile conduisent dans l'ensemble à une bonne adaptation de l'écoulement.

La répartition de vitesse axiale à l'entrée est régulière et correspond donc bien à un écoulement potentiel.

La figure 7 compare les résultats obtenus en aval de la roue mobile.

La répartition de vitesse axiale semble satisfaisante.

Le léger écart apparaissant surtout côté moyeu et un peu côté carter est probablement dû à la couche limite.

Par contre, le calcul conduit à une déviation un peu trop importante de l'écoulement.

L'allure générale des courbes de vitesse tangentielle est la même au milieu de la veine mais un écart plus grand apparait au moyeu et au carter. On peut noter que la prise en compte des couches limites au moyeu et au carter d'une part et du sillage d'autre part,surtout au pied de l'aube, aurait tendance à réduire cet écart. 
Cependant, cet écart étant plus important côté moyeu et les vitesses tangentielles résultantes restant faibles en valeur absolue, la puissance échangée calculée n'est que peu affectée :

$P=52,8 \mathrm{MW}$ après le quatrième calcul méridien.

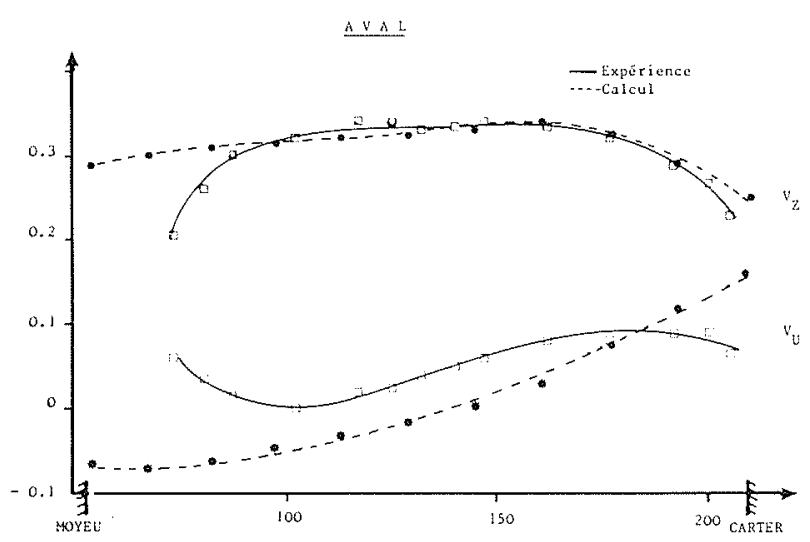

Figure 7 - Comparaison "Calcul-Expérience" à l'aval de la roue mobile.

\section{Etude mécanique}

\section{- Présentation du calcul des contraintes}

Le programme utilisé (CAVIAR) est un code aux éléments finis, en formulation déplacements. Il utilise des éléments isoparamétriques, et permet de traiter des matériaux élastiques ou plastiques.

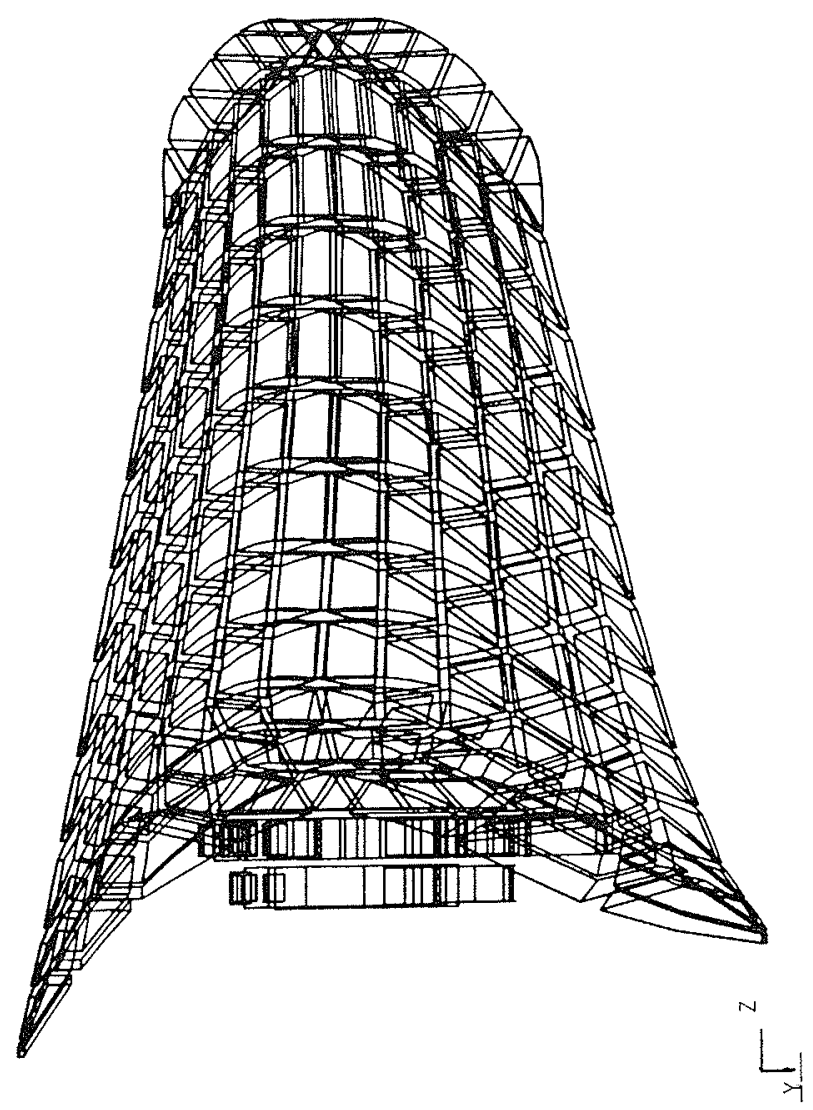

Figure 8 - Maillage de la pale. Vue perpendiculaire à l'axe.

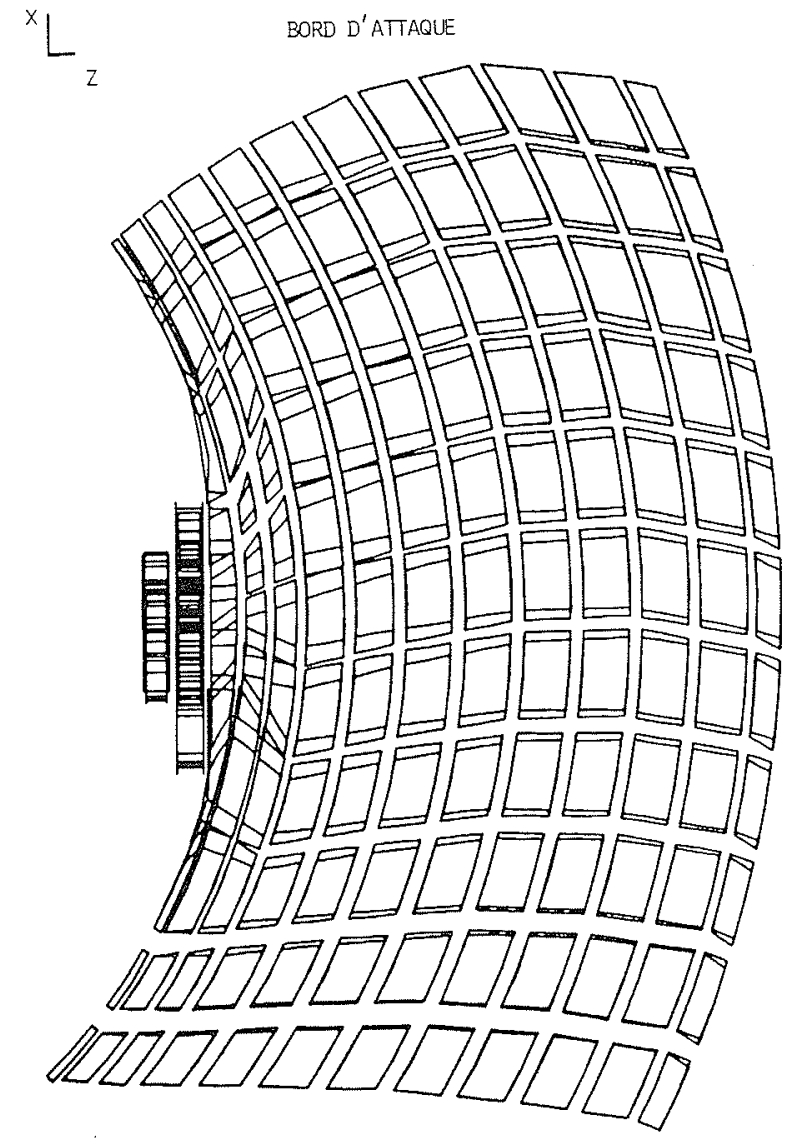

BORD DE FUITE

Figure 9 - Maillage de la pale. Vue suivant l'axe de rotation.

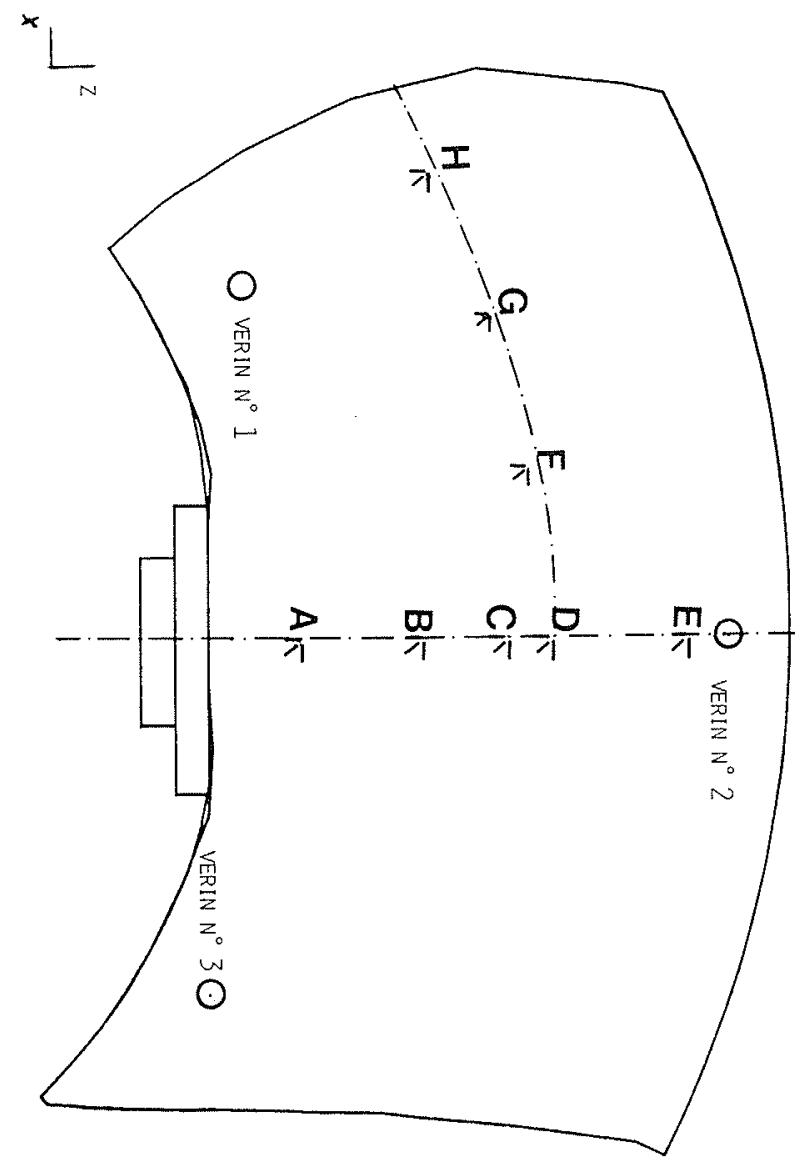

Figure 10 - Emplacement des rosettes et des points d'appui des vérins. 

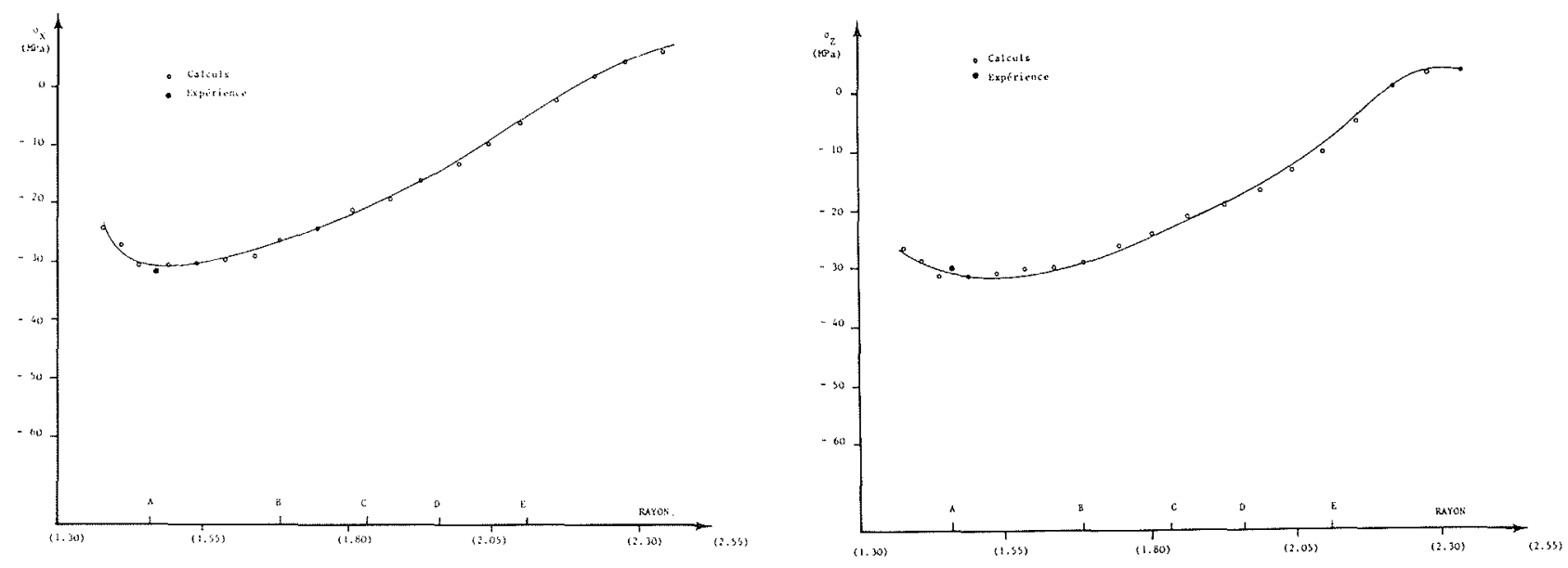

Figure 11 - Premier jeu de vérins (25,4 tonnes, 8 tonnes, 18,4 tonnes) contraintes $\sigma_{x}$ et $\sigma_{z}$.

Dans le cas présent, la pale a été maillée, à l'aide d'un mailleur interactif, en éléments héxaédriques à 20 noeuds et pentahédriques à 15 noeuds, de type Serendip (figs 8 et 9).

Chaque noud comporte trois degrés de liberté (déplacements) et le modèle dans son ensemble comporte 150 éléments pour 1220 nouds, soit 3660 inconnues.

La fixation de la pale est assurée par encastrement des noeuds du bord du tourillon, ce qui est une hypothèse suffisamment représentative des conditions de fonctionnement.

Le matériau est de l'acier, homogène, isotrope, et supposé rester dans le domaine élastique.

Les chargements appliqués à la pale sont, pour la pale sur site :

a) le champ de pression calculé : on applique en fait aux faces d'éléments situés à l'intrados de la pale la différence de pression intrados-extrados.

b) les forces centrifuges dues à la rotation de la pale.

c) le poids propre de la pale,

et pour les essais en atelier :

a) les pressions exercées par les trois verins.

Les champs de contraintes ainsi calculés nous serviront de base aux comparaisons ci-dessous :

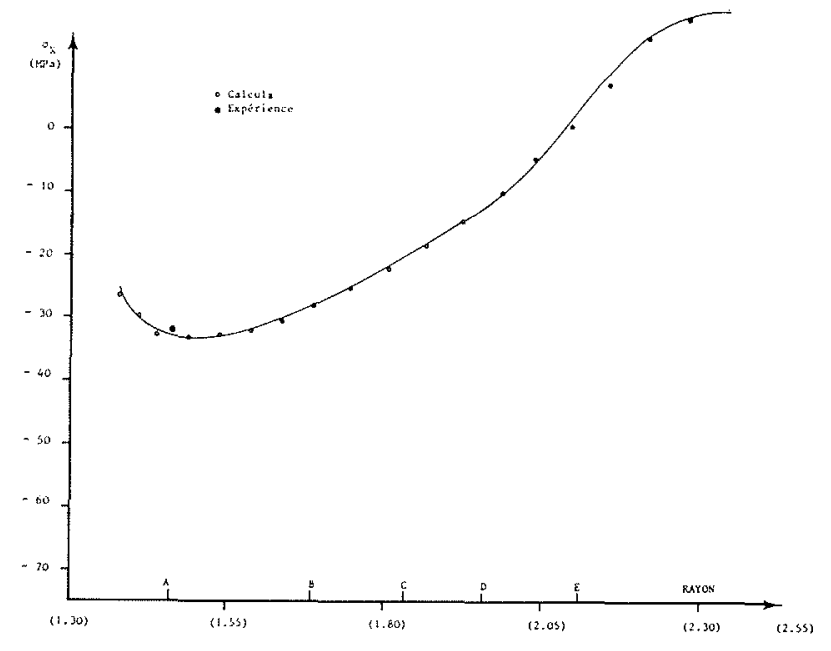

\section{- Les valeurs expérimentales des contraintes}

Deux types de mesures ont été effectués par le Service de la Production Hydraulique d'E.D.F.

\section{a) Mesures sur site [3]}

Ces mesures ont été effectuées sur une pale en fonctionnement réel, pour plusieurs cas de charge.

Pour cela, la pale a été intrumentée côté extrados (partie inférieure de la pale, en compression) par huit "rosettes" (points A à $\mathrm{H}$, voir figure 10) situées d'une part suivant un rayon (rosettes $A$ à $E$ ), d'autre part suivant une ligne correspondant au diamètre hydraulique, côté bord d'attaque (rosettes $D, F, G, H$ ). Ces rosettes étaient reliées à une télémesure transmettant les informations.

Ce sont les contraintes principales en ces points qui seront utilisées pour les comparaisons.

\section{b) Mesures en atelier [4]}

Afin de tester des géométries différentes du congé de raccordement entre la pale et le tourillon, des expériences sur une pale isolée ont été menées en atelier. Pour cela, une pale, montée debout, est soumise à un chargement constitué de trois verins répartis suffisamment loin du tourillon (voir la position des verins sur

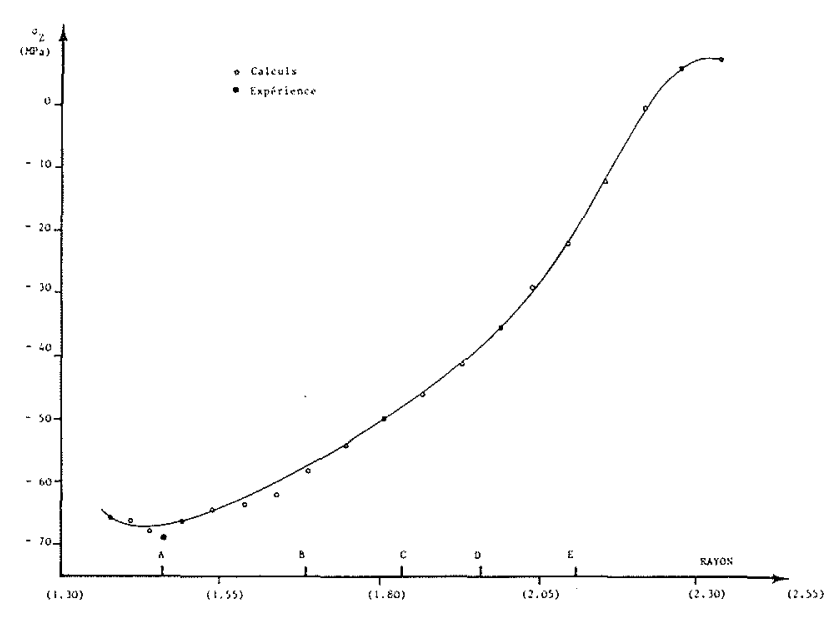

Figure $12-2^{\mathrm{e}}$ jeu de vérins (16 tonnes, 16 tonnes, 12 tonnes) contraintes $\sigma_{x}$ et $\sigma_{z}$. 

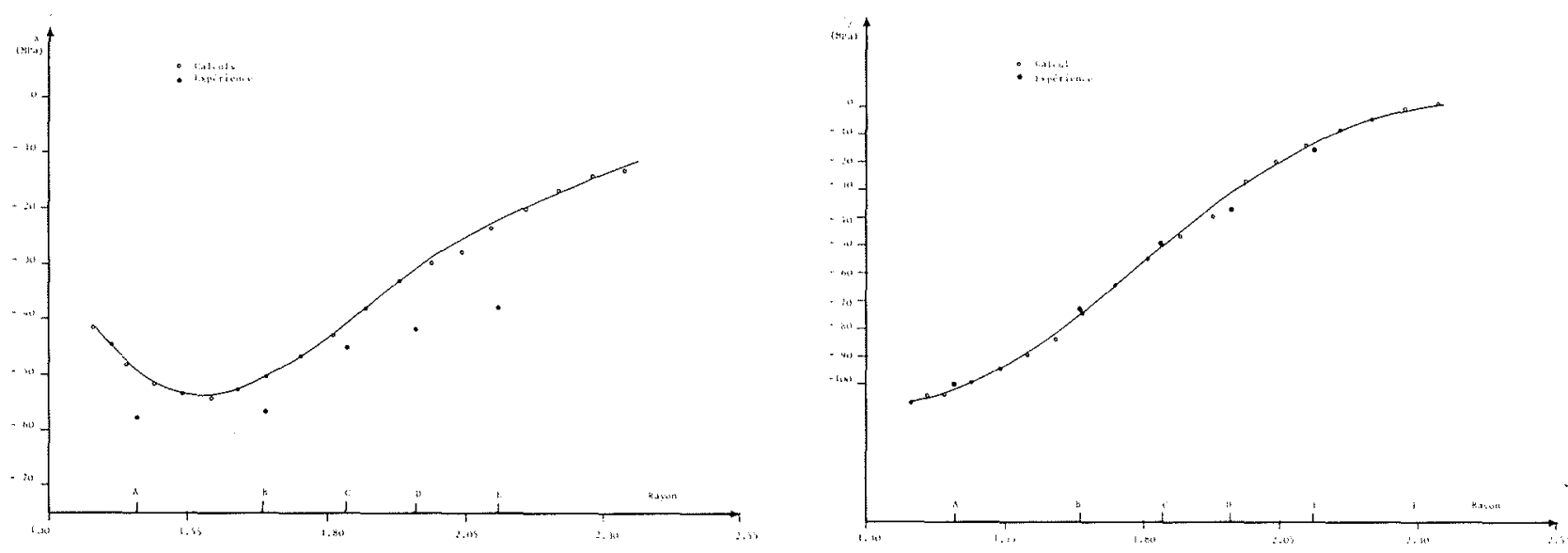

Figure 13 - Machine réelle. Contraintes $\sigma_{x}$ et $\sigma_{z}$. Répartition radiale.

la figure 10) pour ne pas perturber le champ de contrainte au voisinage de celui-ci. Deux chargements ont été effectués, le deuxième étant ajusté pour obtenir un champ de contrainte, au point de raccordement, aussi proche que possible du champ réel en fonctionnement.

Afin de recaler ces valeurs, la rosette $\mathrm{A}$ a été replacée également sur cette pale. Ce sont les contraintes en ces points qui seront examinées ci-dessous.

\section{- Comparaison des résultats théoriques et expérimen- taux}

\section{a) Mesures en atelier}

Les figures 11 et 12 présentent les résultats de répartitions de contraintes $\sigma x$ et $\sigma z$ suivant un rayon. Le seul point de comparaison sur chacune de ces courbes est fourni par la jauge A. Bien que cette comparaison ne porte que sur un point, on peut constater un bon accord entre calculs et expériences.

\section{b) Mesures sur site}

Les figures 13 et 14 présentent les résultats des répartitions de contraintes $\sigma x$ et $\sigma z$, suivant la direction radiale pour la figure 13 et la direction circonférentielle pour la figure 14 .

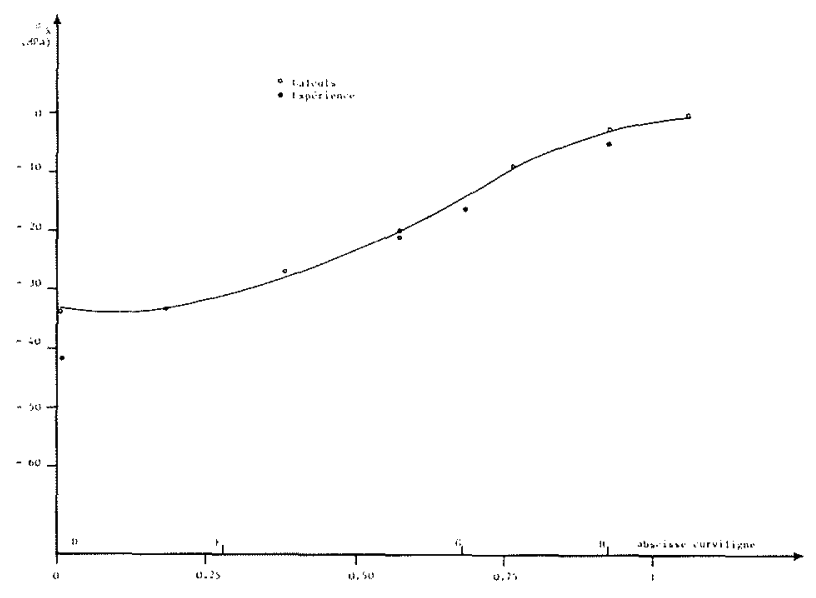

Les valeurs expérimentales relevées, en fonctionnement réel, aux points $\mathrm{A}$ à $\mathrm{H}$ sont reportées sur les mêmes courbes.

Les répartitions de contrainte radiale $\sigma z$ montrent un très bön accord entre les valeurs expérimentales et théoriques. Cette contrainte radiale est représentative de la flexion de la pale soumise aux divers chargements. Une analyse détaillée des contributions de chaque chargement (poids propre, force centrifuge, pression hydrodynamique) montre une très large prépondérance des forces de pression.

Ce bon accord entre expérience et calcul semble donc indiquer une bonne répartition radiale des pressions, donc des puissances échangées, entre le carter et le moyeu.

Les répartitions de contraintes $\sigma x$, aussi bien radiale que circonférentielle, montrent toutefois un plus grand écart entre calcul et expérience ; cet écart résidant à la fois en amplitude (surtout dans la partie centrale de la pale, juste au-dessus du diamètre hydraulique) et en forme.

Cette contrainte est plutôt significative d'une "ouverture" (ou diminution de cambrure) des sections circonférencielles de la pale. Ces différences sembleraient donc montrer que la répartition de pression le long d'une section n'est pas en très bon accord avec la réalité. (Nous pouvons toutefois remarquer que les points de maximum

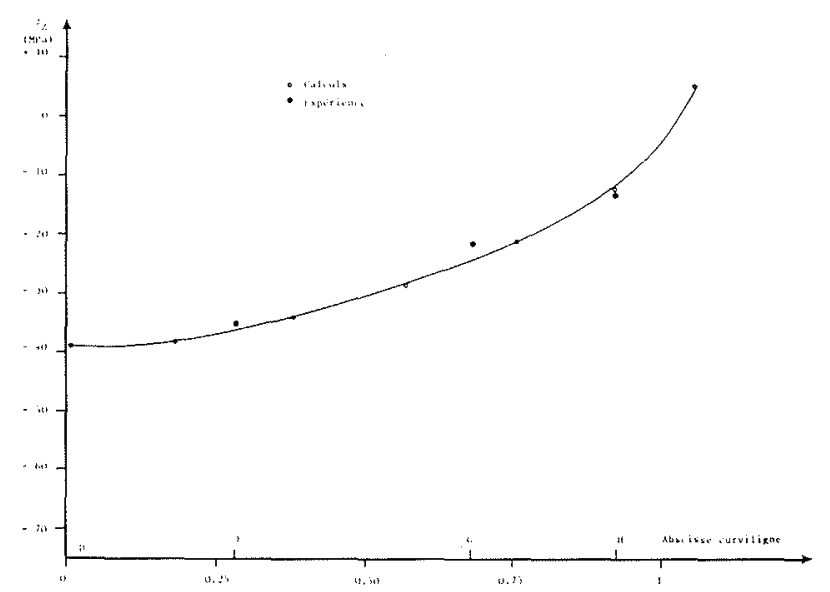

Figure 14 - Machine réelle. Contraintes $\sigma_{x}$ et $\sigma_{z}$. Répartition circonférentielle. 
de contraintes semblent bien mis en évidence). Ce désaccord peut être lié à l'écart observé sur les répartitions de vitesses tangentielles en aval de la roue mobile.

\section{Conclusion}

Les résultats obtenus à l'issue de cette étude sont encourageants : ils montrent que le couplage du calcul d'écoulement et du calcul de contraintes dans une machine hydraulique est possible à l'heure actuelle.

La comparaison calcul-expérience des caractéristiques de l'écoulement souligne cependant la nécessité de prendre en compte les couches limites et les pertes dans le calcul.

En effet, les courbes issues du calcul et de l'expérience coincident en général assez bien au milieu de la veine mais elles laissent toutes apparaître un écart au voisinage du moyeu et du carter, l'allure des vitesses sur les courbes expérimentales au moyeu et au carter s'expliquant par l'existence de couches limites et d'écoulements secondaires, phénomènes qui ne sont pas pris en compte dans le calcul.

Le décalage entre les deux courbes de vitesse tangentielle en aval de la roue peut provenir d'une légère imprécision dans le calcul de l'angle de l'écoulement à la sortie de l'aubage mobile (liée aux diverses interpolations nécessaires à l'application de la condition de KuttaJoukowski). La composante tangentielle de la vitesse étant très sensible à la valeur de l'angle de sortie.

Les résultats de l'étude mécanique montrent un bon accord entre le calcul et l'expérience pour la composante $\sigma_{Z}$ des contraintes; des écarts, probablement liés aux résultats du calcul d'écoulement, apparaissent sur la composante $\sigma_{X}$.
C'est donc dans le sens d'un affinement du calcul d'écoulement qu'il faudra poursuivre cette étude.

\section{Remerciements}

Le présent travail n'a pu être mené à bien que grâce à la collaboration des Ateliers de Constructions Mécaniques des Charmilles (Vevey - Suisse) ${ }^{(1)}$ qui nous ont fourni les données expérimentales relatives à l'écoulement, relevées sur maquette, et grâce à la collaboration du Service de la Production Hydraulique (E.D.F.) qui a effectué les relevés de contraintes sur la machine réelle. Nous remercions vivement toutes les personnes qui ont contribué à ce travail expérimental

\section{Bibliographie}

[1] CHUNG-HUA WU. - A general theory of 3-D flow in subsonic and supersonic turbomachines of axial, radial and mixed flow types. NACA I.Re. 2604 (1952).

[2] KATSANIS T. - A computer program for calculating velocities and streamlines for two dimensional incompressible flow in axial blade rows. NASA TN D-3 762, 1967.

[3] CRAVERO J.M., MASSONAT A. - Usine de Manosque Sainte-Tulle - Mesures de contraintes sur divers congés expérimentaux de raccordement arrière pale-tourillon. Rapport E.D.F./S.P.H. 7746-36 JMC/AM/AMS.

[4] THOMAS P.M. TEHEL J. - Ste Tulle 2 - Manosque Etude de différents congés de raccordement pale-tourillon. Rapport E.D.F./S.P.H. 7759-36 PMT/JT/YA.

(1) Ateliers de Constructions Mécaniques de VEVEY S.A. CH-1800 VEVEY SUISSE

\section{Discussion}

Président : R. BALLAY

Sur intervention de M. TEHEL, M. COMTE précise que le calcul présenté ne permet pas d'évaluer les contraintes au niveau des congés de raccordement pale-tourillon parce que le maillage n'est pas assez raffiné et que la formulation en déplacement utilisée n'assure pas la continuité des contraintes d'un élément à l'autre. Cependant si l'on fait abstraction des difficultés pratiques de réalisation du maillage, un tel calcul ne pose pas de problème. Il a déjà été effectué de manière tout à fait satisfaisante sur d'autres pièces.

Ici le calcul avait surtout pour but de définir un chargement statique représentatif de la charge hydrodynamique pour permettre une optimisation des congés de raccordement paletourillon en atelier.

M. TEHEL ajoute qu'il avait constaté, en effectuant les mesures de contraintes sur les pales de cette machine, des différences de l'ordre de 20 à $30 \%$ entre les pales résultant d'une dispersion du même ordre de grandeur sur les épaisseurs au voisinage des congés de raccordement pale-tourillon. C'est la raison pour laquelle il ne faut pas vouloir aller trop loin dans l'analyse des comparaisons calcul-expérience.

M. CASACCI précise qu'à l'heure actuelle les pales de turbines KAPLAN sont usinées. Il en résulte une grande répétitivité géométrique entre les pales.

En réponse à M. BARIO, Melle ANCONA et M. VERRY précisent que les résultats ont été obtenus après trois itérations entre les surfaces méridiennes et aube à aube, l'écart flux-profil obtenu étant proche des valeurs fournies par les corrélations applicables aux turbines. Il faut toutefois être très prudent car l'incertitude sur la valeur des angles obtenus par application de la condition de KUTTA JOUKOVSKI dans le calcul aube à aube n'est pas négligeable devant les faibles valeurs de l'écart flux-profil existant dans ce type de machine. 


\section{Abstract}

\section{Calculation of the flow field in a Kaplan turbine and of the stresses on the blades}

It was planned to study two aspects of a KAPLAN-type hydraulic turbine:

- a hydrodynamic study to determine the characteristics of the flow,

- a mechanical study to check blade strength.

The flow calculation was made by a quasi-three-dimensional method. (1)

The fluid was incompressible and non-viscous; the flow was stationary; the calculation surfaces were axisymetrical.

Matrix methods based on diagrams of finite differences were used both on the meridian surface and on the blade-toblade surfaces.

The coupling was initiated by a meridian calculation and after the calculations on five blade-to-blade surfaces the average current lines permited the definition of a new meridian surface.

The theoretical and experimental values for the flow characteristics matched well, in the middle of the water-vein, for the axial and tangential velocities upstream of the runner.

Downstream of the runner, the axial-velocity curves matched in the middle of the water-vein but the tangentialvelocity curves differed slightly though they had the same pattern.

Differences appeared at the hub and the casing both upstream and downstream because the viscosity effects were not taken into account in the calculation.
The distributions of static pressure on the blade systems were introduced into the calculation of stresses.

This calculation was made using a finite-elements code, the material being assumed to be homogeneous and elastic in this case.

The forces taken into account were: the pressure load, the centrifugal force, and the weight of the blade itself. The results of these calculations were compared with the experimental values obtained by the Hydraulic Production Department firstly on a blade under actual operating conditions and secondly on a real blade subjected to forces produced by three jacks.

Comparison of the theoretical and experimental results shows a good match for the distribution of radial stresses. However a difference appears between the experimental and theoretical distributions of circumferential stresses. This difference is probably associated with that found for the circumferential velocities.

The results obtained from this study are encouraging: they show that it is possible to couple up calculation of the flow and calculation of the stresses in present-day hydraulic machinery.

The comparison between calculation and experimental concerning the flow characteristics however emphasies the need to take account of the boundary layers and losses in the calculation. 